\title{
An Efficient synthesis of Iminoisoindolione Derivatives by Cobalt-Catalyzed Oxidative Isocyanide Insertion
}

\author{
Feihua Zou, Xuwen Chen, Wenyan Hao*, \\ ${ }^{a}$ College of Chemistry \& Chemical Engineering, Jiangxi Normal University, Nanchang 330022, People's Republic of China
}

\section{ARTICLE INFO}

Article history:

Received

Received in revised form

Accepted

Available online

\section{Keywords:}

Oxidative isocyanide insertion

Cobalt-catalyzed

Iminoisoindoliones

Heterocycles

C-H activation
ABSTRACT

A Cobalt-catayzed oxidative isocyanide insertion of aminoquinoline benzamides has been developed. The reaction proceeded smoothly by using $\mathrm{Ag}_{2} \mathrm{CO}_{3}$ as the oxidant in $\mathrm{PhCl}$ at $120{ }^{\circ} \mathrm{C}$ and delivered products in moderate to excellent yields with excellent functional group tolerance. The transformation is supposed to undergo the ortho C-H activation and isocyanide insertion of C-Co species toward the benzamides. Attractive features of this system include the use of low cost cobalt catalyst, the easyhandling operation, and readily available substrates.

2009 Elsevier Ltd. All rights reserved.
During the past several decades, as a high versatile reagent, isocyanides have wide application in organic, medicinal, and combinatorial chemistry. Indeed, due to its structural and reactive properties, significant achievements have been made in isocyanide-based multicomponent reactions ${ }^{1}$, cycloaddition reactions ${ }^{2}$, heterocycle synthesis ${ }^{3}$ etc. Apart from above reactions, transition-metal-catalyzed isocyanide insertion is another kind of fundamental chemical transformation ${ }^{4}$, which often involved isocyanide as a powerful C1 building blocks to extend the carbon chain length. The classical isocyanide insertion is usually occurred on low-valent transition metal-catalyzed addition of halides to isocyanides, where the oxidative addition and reductive elimination is generally considered happening in the catalytic cycle ${ }^{5}$. However, these methods generally require multistep procedures, not-readily available starting materials, and substrate pre-functionalization. Recently, several examples have been reported via metal-catalyzed intermolecular oxidative isocyanide insertion reaction by means of $\mathrm{C}-\mathrm{H}$ bond activation, which have been emerged as a conceptually new alternative to "classical isocyanide processes". ${ }^{6}$ For example, in 2011, Zhu and co-workers ${ }^{6 a}$ first reported a palladium-catalyzed intramolecular $\mathrm{C}\left(\mathrm{sp}^{2}\right)-\mathrm{H}$ amidination through oxidative isocyanide insertion. This work provides a direct access to 4-aminoquinazolines from $\mathrm{N}$-arylamidines. In 2012, Orru and co-workers ${ }^{6 \mathrm{~b}}$ reported another example of oxidative isocyanide insertion to get diverse cyclic guanidines. But most of these reactions are catalyzed by the noble metal such as palladium and ruthenium. So, further development of non-noble metal catalyzed oxidative isocyanide insertion is highly demanded ${ }^{7}$. Recently, cobalt-catalyzed C-H functionalization has attracted considerable attention due to its economy, low toxicity, and interesting reaction modes ${ }^{8}$. Many $\mathrm{C}-\mathrm{C}^{9}$ and $\mathrm{C}$-heteroatom ${ }^{10}$ bond-forming reactions have been achieved by this method. For example, Murahashi and coworkers reported the first example of a cobalt-catalyzed chelation-assisted C-H functionalization process ${ }^{90}$. In 2014, Daugulis showed an elegant method for the annulation of benzamide using a simple, commercially available cobalt ${ }^{(\mathrm{II})}$ precursor along with a carboxylate base and $\mathrm{Mn}^{(\text {III) }}$ as a cocatalyst under mild conditions $^{11}$. More recently, Miura's group and our group have independently reported copper-catalyzed and nickel-catalyzed insertion reaction of isocyanides with aminoquinoline benzamides to construct Iminoisoindolione (Scheme 1) ) $^{7 \mathrm{a}, 12}$. Although many transition-metal-catalyzed oxidative isocyanide insertion have been reported, the success of direct oxidative isocyanide insertion catalyzed by cobalt is still rare ${ }^{13}$. As our continuation on the development of new catalyst systems for isocyanide chemistry ${ }^{14}$, herein, we report a $\mathrm{Co}(\mathrm{acac})_{2}$-catalyzed isocyanide insertion reaction of benzamides.

1) Miura's work:

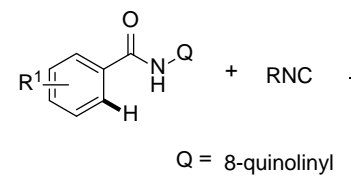

2) Our previous work:
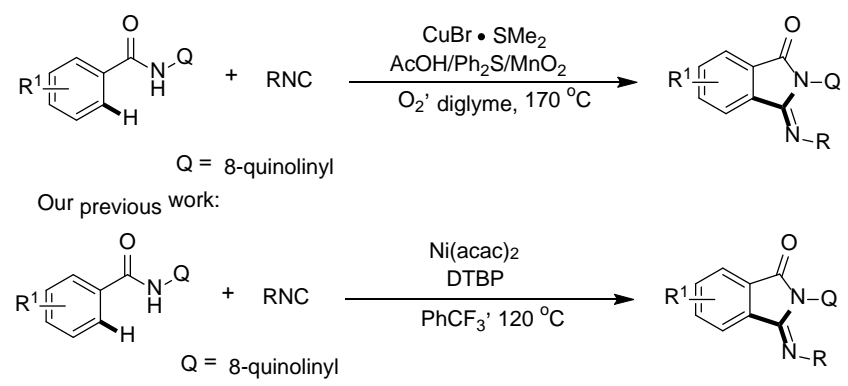

3) This work:

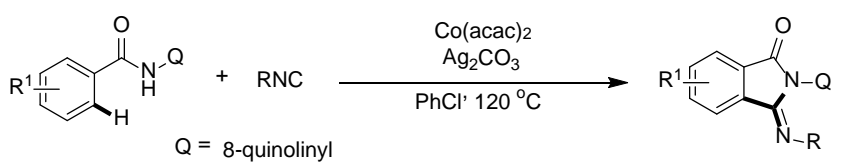

Scheme 1 Recent methods for the synthesis of iminoisoindoliones 
We used benzamide 1a $(0.25 \mathrm{~mol})$ that bears the 8aminoquinoline-based bidentate coordination group and $t$-butyl isocyanide 2a as substrates for the optimization of reaction conditions (Table 1). In the early experiment, the desired iminoisoindolinone 3a was formed in 35\% yield upon treatment of 1a and 2a in the present of a Co(acac) $)_{2}$ catalyst ( $20 \mathrm{~mol} \%$ ) under $\mathrm{O}_{2}$ (1 atm, balloon) in toluene at $120{ }^{\circ} \mathrm{C}$ (Table 1 , entry 1 ). Next, different cobalt salts were screened, but the results were not satisfactory. (Table 1, entries 2-6). Some other oxidants also can promote the reaction and the result indicates the $\mathrm{Ag}_{2} \mathrm{CO}_{3}$ (3.0 eqv.) was the best choice which provided the desired product in 65\% yield (table 1, entries 7-12). Different solvents were screened subsequently (Table 1, entries 13-16). From the results, it seemed that the reaction worked most efficiently when $\mathrm{PhCl}$ was employed as the solvent which provided the desired product in $84 \%$ yield. A lower yield was obtained when the reaction was performed at 140 or $100{ }^{\circ} \mathrm{C}(80 \%, 55 \%$ yield, Table 1 , entries 17 and 18). The reaction cannot proceeded when tempature lower than $80{ }^{\circ} \mathrm{C}$ (Table 1 , entries 19-20).

Table 1 Optimization Studies for Cobalt-Catalyzed Oxidantive Isocyanide Insertion of Benzamide 1 $\mathbf{a}^{a}$<smiles>CC(C)(C)[N+]O[N-]C(=O)c1ccccc1</smiles>

$1 a$

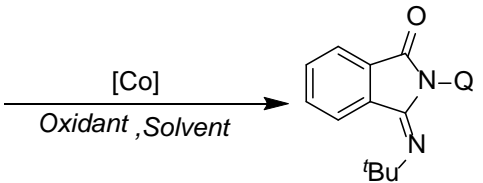

3a

\begin{tabular}{|c|c|c|c|c|c|}
\hline Entry & Catalyst & Oxidant & Solvent & Temp. $\left({ }^{\circ} \mathrm{C}\right)$ & Yield $^{b}(\%)$ \\
\hline 1 & $\mathrm{Co}(\mathrm{acac})_{2}$ & $\mathrm{O}_{2}$ & Toluene & 120 & 35 \\
\hline 2 & $\mathrm{Co}(\mathrm{acac})_{3}$ & $\mathrm{O}_{2}$ & Toluene & 120 & 30 \\
\hline 3 & $\mathrm{CoBr}_{2}$ & $\mathrm{O}_{2}$ & Toluene & 120 & N.D. \\
\hline 4 & $\mathrm{CoCl}_{2}$ & $\mathrm{O}_{2}$ & Toluene & 120 & N.D. \\
\hline 5 & $\mathrm{CoC}_{2} \mathrm{O}_{4}$ & $\mathrm{O}_{2}$ & Toluene & 120 & 30 \\
\hline 6 & $\mathrm{Co}(\mathrm{OAc})_{2} \cdot 4 \mathrm{H}_{2} \mathrm{O}$ & $\mathrm{O}_{2}$ & Toluene & 120 & 15 \\
\hline 7 & $\mathrm{Co}(\mathrm{acac})_{2}$ & TBHP & Toluene & 120 & 30 \\
\hline 8 & $\mathrm{Co}(\text { acac })_{2}$ & DTBP & Toluene & 120 & 35 \\
\hline 9 & $\mathrm{Co}(\mathrm{acac})_{2}$ & $\mathrm{~K}_{2} \mathrm{~S}_{2} \mathrm{O}_{8}$ & Toluene & 120 & trace \\
\hline 10 & $\mathrm{Co}(\mathrm{acac})_{2}$ & $\mathrm{NaIO}_{4}$ & Toluene & 120 & trace \\
\hline 11 & $\mathrm{Co}(\text { acac })_{2}$ & BQ & Toluene & 120 & trace \\
\hline 12 & $\mathrm{Co}(\mathrm{acac})_{2}$ & $\mathrm{Ag}_{2} \mathrm{CO}_{3}$ & Toluene & 120 & 65 \\
\hline 13 & $\mathrm{Co}(\mathrm{acac})_{2}$ & $\mathrm{Ag}_{2} \mathrm{CO}_{3}$ & DMSO & 120 & N.D. \\
\hline 14 & $\mathrm{Co}(\mathrm{acac})_{2}$ & $\mathrm{Ag}_{2} \mathrm{CO}_{3}$ & DCE & 120 & 57 \\
\hline 15 & $\mathrm{Co}(\text { acac })_{2}$ & $\mathrm{Ag}_{2} \mathrm{CO}_{3}$ & $\mathrm{PhCl}$ & 120 & 84 \\
\hline 16 & $\mathrm{Co}(\text { aсас })_{2}$ & $\mathrm{Ag}_{2} \mathrm{CO}_{3}$ & $\mathrm{PhCF}_{3}$ & 120 & 67 \\
\hline 17 & $\mathrm{Co}(\text { acac })_{2}$ & $\mathrm{Ag}_{2} \mathrm{CO}_{3}$ & $\mathrm{PhCl}$ & 140 & 80 \\
\hline 18 & $\mathrm{Co}(\mathrm{acac})_{2}$ & $\mathrm{Ag}_{2} \mathrm{CO}_{3}$ & $\mathrm{PhCl}$ & 100 & 55 \\
\hline 19 & $\mathrm{Co}(\mathrm{acac})_{2}$ & $\mathrm{Ag}_{2} \mathrm{CO}_{3}$ & $\mathrm{PhCl}$ & 80 & N.D. \\
\hline 20 & $\mathrm{Co}(\mathrm{acac})_{2}$ & $\mathrm{Ag}_{2} \mathrm{CO}_{3}$ & $\mathrm{PhCl}$ & r.t. & N.D. \\
\hline
\end{tabular}

TBHP: tert-butyl hydroperoxide, DTBP: di-tert-butyl peroxide, BQ: 1,4-Benzoquinone, DCM: DiChloroMethane. [a]Reaction conditions: Benzamide 1a $(0.25 \mathrm{mmol})$, isocyanide 2a (0.5mmol), $\mathrm{Co}(\mathrm{acac})_{2}(20 \mathrm{~mol} \%), \mathrm{Ag}_{2} \mathrm{CO}_{3}$ (3.0 eqv.) $\mathrm{PhCl}(2 \mathrm{~mL}), 120{ }^{\circ} \mathrm{C}$. [b] Isolated yield based on benzamide $1 \mathrm{a}$.

With this optimized condition (20 mol\% of $\mathrm{Co}(\mathrm{acac})_{2}$, 3.0 equiv.
Table 2 Products in oxidative isocyanide insertion of various benzamides 1 and isocyanides $\mathbf{2}^{\text {[a] }}$
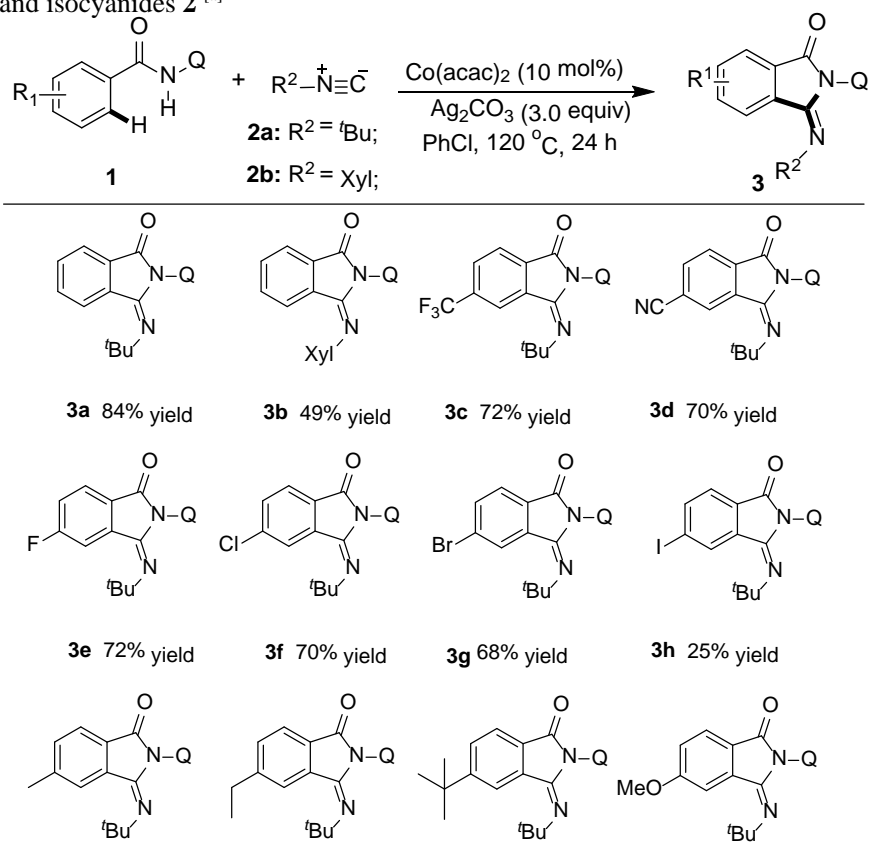<smiles>CC(C)(C)c1ccc2c(c1)/C(=N\C(=O)O)N(O)C2=O</smiles><smiles>COc1ccc2c(c1)/C(=N\C(C)(C)C)N(O)C2=O</smiles><smiles>CC(C)/N=C1\c2cccc(F)c2C(=O)N1OC(C)(C)C</smiles>

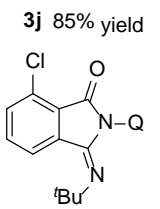<smiles>Cc1cccc2c1C(=O)N(O)/C2=N/C(C)(C)C</smiles>

31 $73 \%$ yield

3m $50 \%$ yield

3n $52 \%$ yield

3o75\% yield

3p 58\% yield<smiles>CC(Br)(Br)/N=C1\c2ccc(F)cc2C(=O)N1O</smiles><smiles>O=C1c2cccc(F)c2/C(=N/CBr)N1O</smiles><smiles>CC(C)/N=C1/c2ccc(Br)cc2C(=O)N1C</smiles><smiles></smiles>

$3 q a$
$72 \%$ yield

$3 q b$

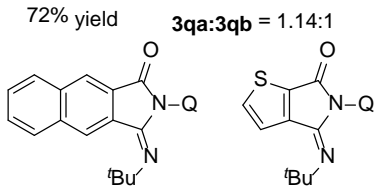

3r $74 \%$ yield

3s $86 \%$
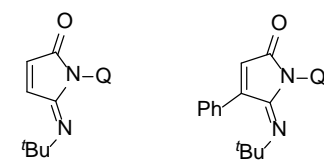

3t $62 \%$ yield

3u $30 \%$ yield

3v N.D.

3w N.D.

[a]Isolated yield based on Benzamide 1a. Q = 8-quinolinyl, Xyl = 2,6-dimethylphenyl. [b] The reaction time was $36 \mathrm{~h}$.

of $\mathrm{Ag}_{2} \mathrm{CO}_{3}, \mathrm{PhCl}, 120{ }^{\circ} \mathrm{C}$ ) in hand, we then explored the scope of the reaction of isocyanides and aminoquinoline benzamides. The results are summarized in Table 2. Various isocyanides were demonstrated to be partners in the transformation. For instance, benzamide 1a reacted with xylyl-substituted isocyanide $\mathbf{2 b}$ leading to the corresponding products $\mathbf{3 b}$ in $49 \%$ yield. In addition to the substrate 1a, electron-withdrawing such as trifluoromethyl, cyano, fluoro, chloro, bromo, iodo groups attached to the aromatic ring at the para position were all tolerated, and the corresponding iminoisoindlinones were obtained in moderate to good yield. The cobalt catalyst also accommodated electron-donating such as methyl, ethyl, tert-butyl and methoxy groups, and they showed somewhat higher efficiency and all have good yields. A similar trend was observed in the case of the substitutent at the congested ortho position. However, to our surprise, the desire product could not be obtained when the methoxy group at ortho position. This may be 
influenced by the steric hindrance effect. Because there are two different reaction sites, two regioisomers should be obtained from the reaction of meta-substituted substrates with isocyanide. However, what is surprising is that the the desired regioisomers of the products was not obtained when bromo, methyl groups attached the aromatic ring at the meta position. The reaction occurred exclusively at the sterically more accessible position, irrespective of electronic nature of the substituent(3r, 3s). Naphthalene was also applicable to this reaction and provided the only product 3t in $62 \%$ yield may be due to the larger steric hindrance effect at 1-position of the naphthalene ring. Moreover, heteroarene such as thiophene-fused iminoisoindolinone was also formed in acceptable yields.

Based on above result and related literature ${ }^{15}$, a proposed mechanism for the reaction of 1a with 2a is illustrated in Scheme 2. $\mathrm{Ag}_{2} \mathrm{CO}_{3}$ may oxidize the $\mathrm{Co}^{\mathrm{II}}$ catalyst to the $\mathrm{Co}^{\mathrm{III}}$ species, which reacts with benzamide (1a) to give the $\mathrm{Co}^{\text {III }}$ intermediate A. Subsequent ortho $\mathrm{C}-\mathrm{H}$ activation produce the intermediate $\mathbf{B}$ followed by the insertion of the isocyanide to the C-Co bond to afford the cobalt ${ }^{(I I)}$ species $\mathbf{C}$. Reductive elimination of the species $\mathbf{C}$ leads to the desired product $3 \mathbf{a}$ and generating the $\mathrm{Co}^{\mathrm{I}}$ catalyst. The latter was oxidized to $\mathrm{Co}^{\mathrm{III}}$ species by $\mathrm{Ag}_{2} \mathrm{CO}_{3}$ again, thus completing the catalytic cycle.

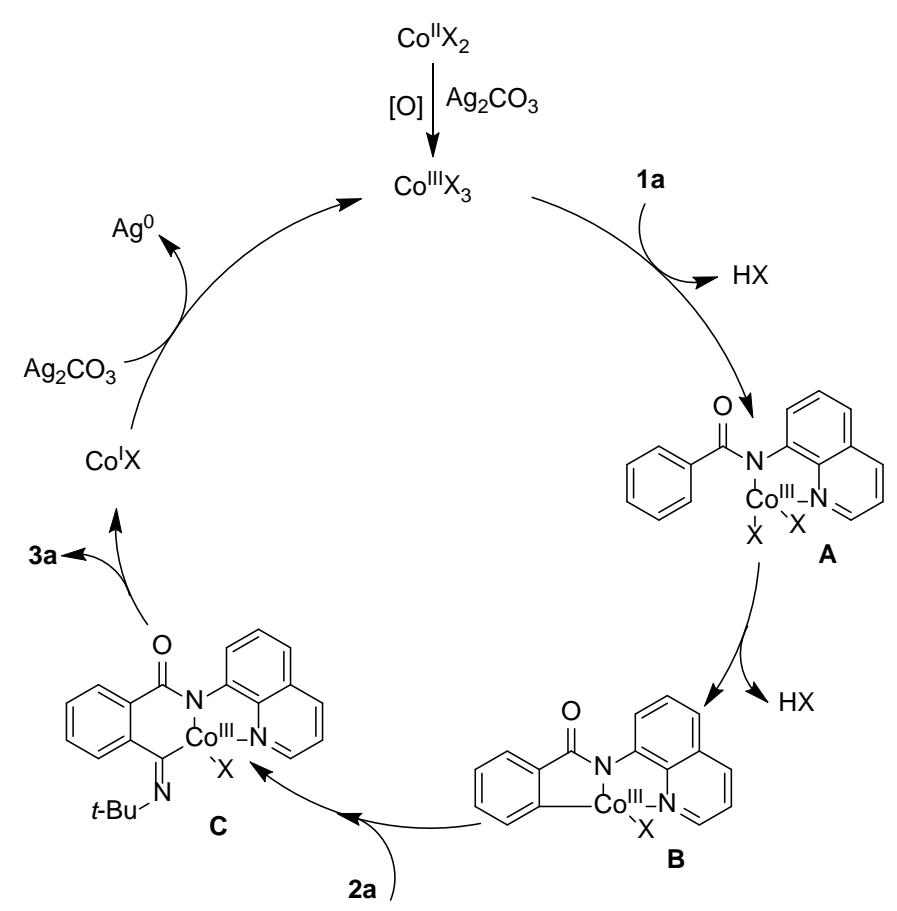

Scheme 2 A plausible mechanism for the cobalt-catalyzed oxidantive ioscyanide insertion with aminoquinoline benzamides

In conclusion, we have developed an effcient cobalt(II)-catalyzed direct oxidative isocyanide insertion through an $\mathrm{sp}^{2} \mathrm{C}-\mathrm{H}$ bond functionalization process. In this reaction, the ability to use isocyanides as $\mathrm{C} 1$ source inserting into $\mathrm{C}-\mathrm{H} / \mathrm{N}-\mathrm{H}$ provides a convenient and alternative route for preparation of iminoisoindolinones in good yields. More transformations incorporating oxidative isocyanide insertion for the synthesis of bioactive heterocycles are currently in progress in our laboratory.

\section{Experimental section}

Synthesis and Characterization for Iminoisoindoliones via Cobalt-Catalyzed Oxidative Isocyanide Insertion

A mixture of benzamide 1 (0.25 mmol) and $\mathrm{Co}(\text { acac })_{2}(0.2$ equiv.) was added into a sealed tube under the $\mathrm{N}_{2}$ atmosphere. Subsequently $\mathrm{PhCl}$ (2 mL), $\mathrm{Ag}_{2} \mathrm{CO}_{3}$ (3.0 equiv.) and isocyanide 2 ( $0.5 \mathrm{mmol}$ ) was added. Then, the sealed tube was heated at 120 ${ }^{\circ} \mathrm{C}$ for 24 hours. After completion of reaction as indicated by TLC, the mixture was concentrated and directly purified by flash column chromatography (EtOAc/petroleum ether, 1:2) to give the desired product 3 .

(E)-3-(tert-butylimino)-2-(quinolin-8-yl)isoindolin-1-one(3a) White solid; (69.2 mg, 84\% ); ${ }^{1} \mathrm{H}$ NMR (400 MHz, $\left.\mathrm{CDCl}_{3}\right) \delta$ 8.85 (dd, $J=1.6,4.0 \mathrm{~Hz}, 1 \mathrm{H}), 8.19$ (dd, $J=1.6,8.4 \mathrm{~Hz}, 1 \mathrm{H}$ ), $8.10(\mathrm{~d}, J=7.6 \mathrm{~Hz}, 1 \mathrm{H}), 8.04(\mathrm{~d}, J=6.8 \mathrm{~Hz}, 1 \mathrm{H}), 7.87$ (dd, $J=$ 1.2, $8.0 \mathrm{~Hz}, 1 \mathrm{H}), 7.61-7.73(\mathrm{~m}, 4 \mathrm{H}), 7.37$ (dd, $J=4.0,8.0 \mathrm{~Hz}$, 1H), 1.37 (s, 9H); ${ }^{13} \mathrm{C}$ NMR (100 MHz, $\left.\mathrm{CDCl}_{3}\right) \delta$ 167.6, 150.2, 147.6, 144.9, 136.1, 134.2, 133.1, 132.3, 131.2, 131.1, 129.7, 129.0, 128.4, 127.2, 125.9, 123.9, 121.2, 53.8, 30.6.

(E)-3-((2,6-dimethylphenyl)imino)-2-(quinolin-8-yl)isoindolin-1one $(3 \boldsymbol{b})$

Black solid; (46.2 mg, 49\% ); ${ }^{1} \mathrm{H}$ NMR (400 MHz, $\mathrm{CDCl}_{3}$ ) $\delta 8.91$ (dd, $J=1.2,4.0 \mathrm{~Hz}, 1 \mathrm{H}$ ), 8.25 (dd, $J=1.2,8.4 \mathrm{~Hz}, 1 \mathrm{H}$ ), 8.00 (dd, $J=7.6,14 \mathrm{~Hz}, 2 \mathrm{H}), 7.95(\mathrm{~d}, J=7.2 \mathrm{~Hz}, 1 \mathrm{H}), 7.76(\mathrm{t}, J=7.6 \mathrm{~Hz}$, $1 \mathrm{H}), 7.64(\mathrm{t}, J=7.2 \mathrm{~Hz}, 1 \mathrm{H}), 7.42-7.48(\mathrm{~m}, 2 \mathrm{H}), 7.06(\mathrm{t}, J=7.0$ $\mathrm{Hz}, 2 \mathrm{H}), 6.99$ (t, $J=7.2 \mathrm{~Hz}, 1 \mathrm{H}), 6.68$ (d, $J=7.6 \mathrm{~Hz}, 1 \mathrm{H}), 2.09$ (s, 3H), 2.02 (s, 3H); ${ }^{13} \mathrm{C}$ NMR (100 MHz, $\left.\mathrm{CDCl}_{3}\right) \delta 168.0$, $152.5,150.8,146.2,144.9,136.3,133.6,132.3,132.2,132.0$, 131.0, 130.6, 129.6, 129.5, 128.3, 128.0, 127.1, 126.6, 126.3, 124.4, 123.9, 123.6, 121.8, 18.2, 18.1.

(E)-3-(tert-butylimino)-2-(quinolin-8-yl)-5-(trifluoromethyl)isoindolin-1-one (3c)

White solid; (71.5 mg, 72\% ); ${ }^{1} \mathrm{H}$ NMR (400 MHz, $\left.\mathrm{CDCl}_{3}\right) \delta$ 8.84 (dd, $J=1.6,4.0 \mathrm{~Hz}, 1 \mathrm{H}), 8.37$ (s, $1 \mathrm{H}), 8.18-8.23$ (m, $2 \mathrm{H})$, 7.98 (d, $J=8.0 \mathrm{~Hz}, 1 \mathrm{H}), 7.92$ (dd, $J=1.2,8.0 \mathrm{~Hz}, 1 \mathrm{H}$ ), 7.74 ( dd, $J=1.6,7.2 \mathrm{~Hz}, 1 \mathrm{H}), 7.67$ (t, $J=8.0 \mathrm{~Hz}, 1 \mathrm{H}), 7.40$ ( dd, $J=8.4$, $4.4 \mathrm{~Hz}, 1 \mathrm{H}), 1.34(\mathrm{~s}, 9 \mathrm{H}) ;{ }^{13} \mathrm{C} \mathrm{NMR}\left(100 \mathrm{MHz}, \mathrm{CDCl}_{3}\right) \delta 166.5$, $150.4,144.7,136.8,136.1,134.7,134.4,1341,133.78,132.8$, 131.1, 129.1, 128.8, 128.3, 128.2, 125.9, 125.0, 124.4, 123.8, 122.3, 121.4, 119.6, 54.1, 30.7; ${ }^{19} \mathrm{~F}$ NMR $\left(\mathrm{CDCl}_{3}, 471 \mathrm{MHz}\right): \delta$ 62.47 .

(E)-3-(tert-butylimino)-1-oxo-2-(quinolin-8-yl)isoindoline-5-carbonitrile (3d)

Yellow solid; (62.0 mg, 70\% ); ${ }^{1} \mathrm{H}$ NMR (400 MHz, $\left.\mathrm{CDCl}_{3}\right) \delta$ 8.83 (dd, $J=1.6,4.0 \mathrm{~Hz}, 1 \mathrm{H}$ ), 8.38 (s, 1H), 8.23 (dd, $J=1.6,8.0$ $\mathrm{Hz}, 1 \mathrm{H}), 8.16$ (d, $J=8.0 \mathrm{~Hz}, 1 \mathrm{H}), 7.99$ (dd, $J=1.2,8.0 \mathrm{~Hz}, 1 \mathrm{H}$ ), 7.93 (dd, $J=1.6,8.0 \mathrm{~Hz}, 1 \mathrm{H}), 7.65-7.74$ (m, 2H), 7.42 (dd, $J=$ 4.0, 8.0 Hz, 1H), $1.32(\mathrm{~s}, 9 \mathrm{H}) ;{ }^{13} \mathrm{C}$ NMR (100 MHz, $\left.\mathrm{CDCl}_{3}\right) \delta$ 166.0, 150.4, 144.8, 144.6, 137.1, 136.2, 134.8, 132.6, 131.1, 130.3, 129.1, 128.9, 125.9, 124.6, 121.5, 118.1, 116.0, 54.3, 30.8.

(E)-3-(tert-butylimino)-5-fluoro-2-(quinolin-8-yl)isoindolin-1-on$e(3 e)$

White solid; (62.5 mg, 72\% ); ${ }^{1} \mathrm{H}$ NMR (400 $\left.\mathrm{MHz}, \mathrm{CDCl}_{3}\right) \delta$ 8.85 (dd, $J=1.6,4.0 \mathrm{~Hz}, 1 \mathrm{H}$ ), 8.21 (dd, $J=1.6,8.4 \mathrm{~Hz}, 1 \mathrm{H}$ ), 8.05 (dd, $J=5.2,8.4 \mathrm{~Hz}, 1 \mathrm{H}), 7.90$ (dd, $J=1.2,8.0 \mathrm{~Hz}, 1 \mathrm{H}$ ), 7.80 (dd, $J=2.0,9.2 \mathrm{~Hz}, 1 \mathrm{H}), 7.72$ (dd, $J=1.2,6.8 \mathrm{~Hz}, 1 \mathrm{H}), 7.66$ (t, $J$ $=7.8 \mathrm{~Hz}, 1 \mathrm{H}), 7.36-7.42(\mathrm{~m}, 2 \mathrm{H}), 1.33(\mathrm{~s}, 9 \mathrm{H}) ;{ }^{13} \mathrm{C}$ NMR $(100$ $\left.\mathrm{MHz}, \mathrm{CDCl}_{3}\right) \delta 166.8,165.2\left(\mathrm{~d},{ }^{1} J_{\mathrm{CF}}=250 \mathrm{~Hz}\right), 150.3,144.8$, 136.1, 133.0, 131.2, 129.9, 129.1, 128.6, 125.9, 125.8, 121.3, $118.3\left(\mathrm{~d},{ }^{2} J_{\mathrm{CF}}=23 \mathrm{~Hz}\right), 114.6\left(\mathrm{~d},{ }^{2} J_{\mathrm{CF}}=25 \mathrm{~Hz}\right), 53.9,30.7$.

(E)-3-(tert-butylimino)-5-chloro-2-(quinolin-8-yl)isoindolin-1-one $(3 f)$ 
Yellow solid; (63.7 mg, 70\% ); ${ }^{1} \mathrm{H}$ NMR (400 MHz, $\left.\mathrm{CDCl}_{3}\right) \delta$ 8.85 (dd, $J=1.6,4.0 \mathrm{~Hz}, 1 \mathrm{H}$ ), 8.21 (dd, $J=1.6,8.4 \mathrm{~Hz}, 1 \mathrm{H}$ ), 8.08 (d, $J=1.6 \mathrm{~Hz}, 1 \mathrm{H}), 8.00$ (d, $J=8.0 \mathrm{~Hz}, 1 \mathrm{H}$ ), 7.91 (dd, $J=$ 1.6, $8.0 \mathrm{~Hz}, 1 \mathrm{H}), 7.64-7.73(\mathrm{~m}, 3 \mathrm{H}), 7.40$ (dd, $J=4.0,8.0 \mathrm{~Hz}$, $1 \mathrm{H}), 1.33(\mathrm{~s}, 9 \mathrm{H}) ;{ }^{13} \mathrm{C}$ NMR $\left(100 \mathrm{MHz}, \mathrm{CDCl}_{3}\right) \delta 166.8,150.3$, 145.9 , 144.8, 138.7, 136.1, 133.0, 132.2, 131.3, 131.2, 129.0, 128.6, 127.2, 125.9, 125.0, 121.3, 54.0, 30.7;

(E)-5-bromo-3-(tert-butylimino)-2-(quinolin-8-yl)isoindolin-1one $(3 \mathrm{~g})$

Yellow solid; (69.4 mg, 68\% ); ${ }^{1} \mathrm{H}$ NMR (400 MHz, $\left.\mathrm{CDCl}_{3}\right) \delta$ 8.84 (dd, $J=1.6,4.0 \mathrm{~Hz}, 1 \mathrm{H}$ ), 8.24 (d, $J=1.6 \mathrm{~Hz}, 1 \mathrm{H}$ ), 8.21 (dd, $J=1.6,8.0 \mathrm{~Hz}, 1 \mathrm{H}$ ), 7.89-7.94 (m, 2H), 7.84 (dd, $J=1.2,8.0 \mathrm{~Hz}$, $1 \mathrm{H}), 7.72$ (dd, $J=1.2,7.2 \mathrm{~Hz}, 1 \mathrm{H}), 7.66(\mathrm{t}, J=7.6 \mathrm{~Hz}, 1 \mathrm{H}), 7.40$ (dd, $J=4.0,8.4 \mathrm{~Hz}, 1 \mathrm{H}), 1.33(\mathrm{~s}, 9 \mathrm{H}) ;{ }^{13} \mathrm{C}$ NMR $(100 \mathrm{MHz}$, $\left.\mathrm{CDCl}_{3}\right) \delta 166.9,150.3,145.7,144.8,136.1,134.2,133.0,131.2$, 130.0, 129.0, 128.6, 127.0, 125.9, 125.2, 121.3, 54.0, 30.7.

(E)-3-(tert-butylimino)-5-iodo-2-(quinolin-8-yl)isoindolin-1-on- $e$ (3h)

Yellow solid. (28.5 mg, 25\% ); ${ }^{1} \mathrm{H}$ NMR (400 MHz, $\left.\mathrm{CDCl}_{3}\right) \delta$ 8.84 (dd, $J=1.6,4.0 \mathrm{~Hz}, 1 \mathrm{H}$ ), 8.45 (s, $1 \mathrm{H}), 8.22$ (dd, $J=1.6,8.4$ $\mathrm{Hz}, 1 \mathrm{H}$ ), 8.05 (dd, $J=1.2,8.0 \mathrm{~Hz}, 1 \mathrm{H}$ ), 7.91 (dd, $J=1.2,8.0 \mathrm{~Hz}$, $1 \mathrm{H}), 7.79$ (d, $J=8.0 \mathrm{~Hz}, 1 \mathrm{H}), 7.71$ (dd, $J=1.2,7.2 \mathrm{~Hz}, 1 \mathrm{H}), 7.66$ (t, $J=7.6 \mathrm{~Hz}, 1 \mathrm{H}), 7.41$ (dd, $J=4.0,8.0 \mathrm{~Hz}, 1 \mathrm{H}), 1.32(\mathrm{~s}, 9 \mathrm{H})$; ${ }^{13} \mathrm{C}$ NMR (100 MHz, $\left.\mathrm{CDCl}_{3}\right) \delta 167.2,150.3,144.8,140.1,136.1$, 135.9, 133.2, 132.9, 131.2, 129.0, 128.6, 125.9, 125.3, 121.3, 54.0, 30.7.

(E)-3-(tert-butylimino)-5-methyl-2-(quinolin-8-yl)isoindolin-1-on e (3i)

Yellow solid; (69.5 mg, 81\% ); ${ }^{1} \mathrm{H}$ NMR (400 MHz, $\mathrm{CDCl}_{3}$ ) $\delta$ 8.82 (dd, $J=1.6,4.0 \mathrm{~Hz}, 1 \mathrm{H}$ ), 8.16 (dd, $J=1.6,8.4 \mathrm{~Hz}, 1 \mathrm{H}$ ), 7.91 (d, $J=9.6 \mathrm{~Hz}, 1 \mathrm{H}), 7.84-7.87$ (m, 2H), 7.69 (dd, $J=5.88$, 1.2, $7.2 \mathrm{~Hz}, 1 \mathrm{H}), 7.62$ (t, $J=7.8 \mathrm{~Hz}, 1 \mathrm{H}), 7.46$ (d, $J=7.6 \mathrm{~Hz}$, $1 \mathrm{H}), 7.35$ (dd, $J=4.0,8.4 \mathrm{~Hz}, 1 \mathrm{H}), 2.56(\mathrm{~s}, 3 \mathrm{H}), 1.32(\mathrm{~s}, 9 \mathrm{H}) ;{ }^{13} \mathrm{C}$ NMR $\left(100 \mathrm{MHz}, \mathrm{CDCl}_{3}\right) \delta 167.7,150.2,147.6,145.0,142.9$, $136.0,133.3,131.8,131.7,131.2,130.3,129.0,128.3,127.8$, 125.9, 123.7, 121.2, 53.7, 30.6, 22.4.

(E)-3-(tert-butylimino)-5-ethyl-2-(quinolin-8-yl)isoindolin-1-one (3j)

Yellow solid. (75.9 mg, 85\% ); ${ }^{1} \mathrm{H}$ NMR (400 $\mathrm{MHz}, \mathrm{CDCl}_{3}$ ) $\delta$ 8.86 (dd, $J=1.2,4.0 \mathrm{~Hz}, 1 \mathrm{H}), 8.19$ (d, $\mathrm{J}=8.4 \mathrm{~Hz}, 1 \mathrm{H}), 7.96$ (t, $J$ $=8.8 \mathrm{~Hz}, 2 \mathrm{H}), 7.88(\mathrm{~d}, J=8.0 \mathrm{~Hz}, 1 \mathrm{H}), 7.72(\mathrm{dd}, J=1.2,7.2 \mathrm{~Hz}$, $1 \mathrm{H}), 7.65$ (t, $J=7.8 \mathrm{~Hz}, 1 \mathrm{H}), 7.52$ (d, $J=7.6 \mathrm{~Hz}, 1 \mathrm{H}), 7.38$ (dd, $J$ $=4.0,8.4 \mathrm{~Hz}, 1 \mathrm{H}), 2.88(\mathrm{q}, J=7.6 \mathrm{~Hz}, 2 \mathrm{H}), 1.37(\mathrm{~s}, 9 \mathrm{H}), 1.37(\mathrm{t}$, $J=7.6 \mathrm{~Hz}, 3 \mathrm{H}) .{ }^{13} \mathrm{C}$ NMR $\left(100 \mathrm{MHz}, \mathrm{CDCl}_{3}\right) \delta 167.7,150.2$, $149.3,147.8,145.0,136.0,133.3,131.9,131.2,130.8,129.0$, 128.3, 126.8, 125.9, 123.8, 121.2, 53.7, 30.6, 29.7, 15.8. HRMS calcd for $\mathrm{C}_{23} \mathrm{H}_{24} \mathrm{~N}_{3} \mathrm{O}^{+}\left(\mathrm{M}+\mathrm{H}^{+}\right)$: 358.1914; Found: 358.1910.

(E)-5-(tert-butyl)-3-(tert-butylimino)-2-(quinolin-8-yl)isoindolin1-one (3k)

Yellow solid; (75.2 mg, 78\% ); ${ }^{1} \mathrm{H}$ NMR (400 MHz, $\left.\mathrm{CDCl}_{3}\right) \delta$ 8.86 (dd, $J=1.6,4.0 \mathrm{~Hz}, 1 \mathrm{H}), 8.18-8.21(\mathrm{~m}, 2 \mathrm{H}), 7.99$ (d, $J=$ $8.0 \mathrm{~Hz}, 1 \mathrm{H}), 7.88(\mathrm{dd}, J=1.2,8.0 \mathrm{~Hz}, 1 \mathrm{H}), 7.71-7.74(\mathrm{~m}, 2 \mathrm{H})$, 7.65 (t, $J=7.6 \mathrm{~Hz}, 1 \mathrm{H}), 7.39$ (dd, $J=4.0,8.4 \mathrm{~Hz}, 1 \mathrm{H}), 1.46$ (s, 9H), 1.39 (s, 9H); 13C NMR (100 MHz, $\left.\mathrm{CDCl}_{3}\right) \delta 167.7,156.1$, 150.2 , 145.0, 136.0, 133.3, 131.6, 131.2, 129.0, 128.3, 128.2, 127.4, 125.9, 124.6, 123.5, 121.2, 116.4, 53.6, 35.7, 31.4, 30.6.

(E)-3-(tert-butylimino)-5-methoxy-2-(quinolin-8-yl)isoindolin-1one (3I)

Yellow solid; (65.6 mg, 73\% ); ${ }^{1} \mathrm{H}$ NMR (400 $\left.\mathrm{MHz}, \mathrm{CDCl}_{3}\right) \delta$ 8.82 (dd, $J=1.6,4.4 \mathrm{~Hz}, 1 \mathrm{H}), 8.17$ (dd, $J=1.6,8.0 \mathrm{~Hz}, 1 \mathrm{H}$ ),
7.95 (d, $J=8.0 \mathrm{~Hz}, 1 \mathrm{H}$ ), 7.85 (dd, $J=1.2,8.0 \mathrm{~Hz}, 1 \mathrm{H}$ ), 7.69 (dd, $J=1.2,7.2 \mathrm{~Hz}, 1 \mathrm{H}), 7.58-7.64(\mathrm{~m}, 2 \mathrm{H}), 7.36$ (dd, $J=4.0,8.0 \mathrm{~Hz}$, $1 \mathrm{H}), 7.14$ (dd, $J=2.4,8.4 \mathrm{~Hz}, 1 \mathrm{H}), 3.94$ (s, 3H), 1.31 (s, 9H); ${ }^{13} \mathrm{C}$ NMR $\left(100 \mathrm{MHz}, \mathrm{CDCl}_{3}\right) \delta 167.6,163.1,150.2,147.1,145.0$, 136.0, 133.4, 131.3, 129.0, 128.3, 126.5, 125.9, 125.3, 121.2, 115.7, 113.8, 56.0, 53.6, 30.6.

(E)-3-(tert-butylimino)-7-fluoro-2-(quinolin-8-yl)isoindolin-1one (3m)

Yellow solid; (43.4 mg, 50\% ); ${ }^{1} \mathrm{H}$ NMR (400 MHz, $\left.\mathrm{CDCl}_{3}\right) \delta$ 8.84 (dd, $J=1.6,4.0 \mathrm{~Hz}, 1 \mathrm{H}$ ), 8.19 (dd, $J=1.6,8.4 \mathrm{~Hz}, 1 \mathrm{H}$ ), 7.87-7.91 (m, 2H), 7.69-7.74 (m, 2H), $7.63(\mathrm{t}, J=7.8 \mathrm{~Hz}, 1 \mathrm{H})$, 7.38 (dd, $J=4.4,8.4 \mathrm{~Hz}, 1 \mathrm{H}), 7.33$ (t, $J=8.6 \mathrm{~Hz}, 1 \mathrm{H}), 1.29$ (s, 9H); ${ }^{13} \mathrm{C}$ NMR $\left(100 \mathrm{MHz}, \mathrm{CDCl}_{3}\right) \delta 164.6,158.6\left(\mathrm{~d},{ }^{1} J_{\mathrm{CF}}=262\right.$ $\mathrm{Hz}), 150.3,145.9,144.9,136.1,134.3\left(\mathrm{~d},{ }^{3} J_{\mathrm{CF}}=8 \mathrm{~Hz}\right), 132.9$, 131.3, 129.0, 128.6, 123.1, 121.3, $119.2\left(\mathrm{~d},{ }^{2} J_{\mathrm{CF}}=19 \mathrm{~Hz}\right), 53.9$, 30.5 .

(E)-3-(tert-butylimino)-7-chloro-2-(quinolin-8-yl)isoindolin-1one (3n)

Yellow solid; (47.3 mg, 52\% ); ${ }^{1} \mathrm{H}$ NMR (400 MHz, $\left.\mathrm{CDCl}_{3}\right) \delta$ 8.86 (dd, $J=1.6,4.0 \mathrm{~Hz}, 1 \mathrm{H}), 8.21(\mathrm{dd}, J=1.6,8.4 \mathrm{~Hz}, 1 \mathrm{H})$, 8.13 (d, $J=7.6 \mathrm{~Hz}, 1 \mathrm{H}), 8.07$ (d, $J=6.8 \mathrm{~Hz}, 1 \mathrm{H}), 7.90$ (dd, $J=$ 1.2, $8.0 \mathrm{~Hz}, 1 \mathrm{H}), 7.64-7.77$ (m, 4H), 7.39 (dd, $J=4.0,8.4 \mathrm{~Hz}$, 1H), 1.37 (s, 9H); ${ }^{13} \mathrm{C}$ NMR (100 MHz, $\left.\mathrm{CDCl}_{3}\right) \delta$ 167.6, 150.2, 147.6, 144.9, 136.1, 134.2, 133.1, 132.3, 131.2, 131.1, 129.0, 128.4, 127.2, 125.9, 123.9, 121.2, 53.8, 30.6; HRMS calcd for $\mathrm{C}_{21} \mathrm{H}_{19} \mathrm{ClN}_{3} \mathrm{O}^{+}\left(\mathrm{M}+\mathrm{H}^{+}\right)$: 364.1211; Found: 364.1201.

(E)-3-(tert-butylimino)-7-methyl-2-(quinolin-8-yl)isoindolin-1-one (3o)

Yellow solid; (64.4 mg, 75\% ); ${ }^{1} \mathrm{H}$ NMR (400 MHz, $\left.\mathrm{CDCl}_{3}\right) \delta$ 8.88 (d, $J=4.0 \mathrm{~Hz}, 1 \mathrm{H}), 8.20$ (d, $J=8.4 \mathrm{~Hz}, 1 \mathrm{H}), 7.97$ (d, $J=7.6$ Hz, 1H), 7.88 (d, $J=8.0 \mathrm{~Hz}, 1 \mathrm{H}), 7.72$ (d, $J=7.2 \mathrm{~Hz}, 1 \mathrm{H}), 7.66$ $(\mathrm{t}, J=7.6 \mathrm{~Hz}, 1 \mathrm{H}), 7.59(\mathrm{t}, J=7.8 \mathrm{~Hz}, 1 \mathrm{H}), 7.43(\mathrm{~d}, J=7.6 \mathrm{~Hz}$, $1 \mathrm{H}), 7.39$ (dd, $J=4.0,8.4 \mathrm{~Hz}, 1 \mathrm{H}), 2.82$ (s, 3H), 1.35 (s, 9H); ${ }^{13} \mathrm{C}$ NMR $\left(100 \mathrm{MHz}, \mathrm{CDCl}_{3}\right) \delta 168.5,150.3,147.3,145.1,138.3$, 136.1, 133.6, 133.4, 131.8, 131.3, 130.7, 129.0, 128.3, 125.9, 124.9, 121.2, 53.6, 30.6, 18.2.

(E)-3-(tert-butylimino)-4,6-dimethyl-2-(quinolin-8-yl)isoindolin1-one $(3 p)$

Yellow solid; (51.8 mg, 58\% ); ${ }^{1} \mathrm{H}$ NMR (400 MHz, $\mathrm{CDCl}_{3}$ ) $\delta$ 8.87 (dd, $J=1.6,4.0 \mathrm{~Hz}, 1 \mathrm{H}$ ), 8.21 (dd, $J=1.6,6.8 \mathrm{~Hz}, 1 \mathrm{H}$ ), 7.95 (dd, $J=1.2,8.4 \mathrm{~Hz}, 1 \mathrm{H}$ ), 7.83 (dd, $J=1.2,7.6 \mathrm{~Hz}, 1 \mathrm{H}$ ), 7.69 (t, $J$ $=7.6 \mathrm{~Hz}, 1 \mathrm{H}), 7.57(\mathrm{~s}, 1 \mathrm{H}), 7.42(\mathrm{dd}, J=3.6,8.0 \mathrm{~Hz}, 1 \mathrm{H}), 7.30$ (d, $J=6.0 \mathrm{~Hz}, 2 \mathrm{H}), 2.74(\mathrm{~s}, 3 \mathrm{H}), 2.47$ (s, 3H), $0.82(\mathrm{~s}, 9 \mathrm{H}) ;{ }^{13} \mathrm{C}$ NMR $\left(100 \mathrm{MHz}, \mathrm{CDCl}_{3}\right) \delta 171.8,151.1,148.2,146.0,141.3$, 140.1, 137.3, 136.7, 136.4, 136.2, 136.1, 133.2, 132.1, 130.5, 129.2, 125.9, 121.8, 121.1, 54.9, 31.5, 21.5, 19.8. HRMS calcd for $\mathrm{C}_{23} \mathrm{H}_{24} \mathrm{~N}_{3} \mathrm{O}^{+}\left(\mathrm{M}+\mathrm{H}^{+}\right)$: 358.1914; Found: 358.1909.

(E)-3-(tert-butylimino)-6-fluoro-2-(quinolin-8-yl)isoindolin-1one (3qa)

Yellow solid; (33.0 mg, 38\% ); ${ }^{1} \mathrm{H}$ NMR (400 MHz, $\left.\mathrm{CDCl}_{3}\right) \delta$ 8.85 (d, $J=4.0 \mathrm{~Hz}, 1 \mathrm{H}$ ), 8.20 (d, $J=8.0 \mathrm{~Hz}, 1 \mathrm{H}$ ), 8.11 (dd, $J=$ $4.4 \mathrm{~Hz}, 8.4,1 \mathrm{H}), 7.89$ (d, $J=8.0 \mathrm{~Hz}, 1 \mathrm{H}), 7.71-7.74(\mathrm{~m}, 2 \mathrm{H})$, $7.65(\mathrm{t}, J=7.8 \mathrm{~Hz}, 1 \mathrm{H}), 7.38-7.40(\mathrm{~m}, 2 \mathrm{H}), 1.35(\mathrm{~s}, 9 \mathrm{H}) ;{ }^{13} \mathrm{C}$ NMR (100 MHz, CDCl $)_{3} \delta 166.4,164.2\left(\mathrm{~d},{ }^{1} J_{\mathrm{CF}}=253 \mathrm{~Hz}\right), 150.3$, 146.5, 144.8, 137.0(d, $\left.{ }^{3} J_{\mathrm{CF}}=8 \mathrm{~Hz}\right), 136.1,132.8,131.1,129.2(\mathrm{~d}$, $\left.{ }^{3} J_{\mathrm{CF}}=8 \mathrm{~Hz}\right), 129.0,128.6,125.9,125.7,121.3,119.4\left(\mathrm{~d},{ }^{2} J_{\mathrm{CF}}=\right.$ 23Hz), $110.9\left(\mathrm{~d},{ }^{2} J_{\mathrm{CF}}=23 \mathrm{~Hz}\right), 53.8,30.6$.

(E)-3-(tert-butylimino)-4-fluoro-2-(quinolin-8-yl)isoindolin-1one $(3 q \boldsymbol{b})$ 
Yellow solid; (29.5 mg, 34\% ); ${ }^{1} \mathrm{H}$ NMR (400 MHz, $\mathrm{CDCl}_{3}$ ) $\delta$ 8.86 (d, $J=4.0 \mathrm{~Hz}, 1 \mathrm{H}), 8.23$ (d, $J=8.4 \mathrm{~Hz}, 1 \mathrm{H}), 7.93$ (d, $J=8.4$ Hz, 1H), 7.88 (d, $J=7.6 \mathrm{~Hz}, 1 \mathrm{H}), 7.66-7.75$ (m, 3H), 7.40-7.45 (m, 2H), 1.16 (s, 9H); ${ }^{13} \mathrm{C}$ NMR (100 $\left.\mathrm{MHz} \mathrm{CDCl}_{3}\right) \delta 167.2$, $155.8\left(\mathrm{~d},{ }^{1} J_{\mathrm{CF}}=258 \mathrm{~Hz}\right), 150.5,145.0,141.2,136.1,135.8$, 133.7, 133.6, 131.3, 129.0, 128.7, 125.9, 121.7 (d, $\left.{ }^{2} J_{\mathrm{CF}}=25 \mathrm{~Hz}\right)$, 121.4, $119.9\left(\mathrm{~d},{ }^{3} J_{\mathrm{CF}}=4 \mathrm{~Hz}\right), 54.8,31.2$.

(E)-6-bromo-3-(tert-butylimino)-2-(quinolin-8-yl)isoindolin-1-o ne (3r)

Yellow solid; (75.5 mg, 74\% ); ${ }^{1} \mathrm{H}$ NMR (400 MHz, $\mathrm{CDCl}_{3}$ ) $\delta$ 8.84 (dd, $J=1.6,2.4 \mathrm{~Hz}, 1 \mathrm{H}$ ), 8.19-8.21 (m, 2H), 7.98 (d, $J=8.4$ $\mathrm{Hz}, 1 \mathrm{H}), 7.85-9.91(\mathrm{~m}, 2 \mathrm{H}), 7.72$ (dd, $J=1.6,6.0 \mathrm{~Hz}, 1 \mathrm{H}), 7.65$ (t, $J=7.8 \mathrm{~Hz}, 1 \mathrm{H}), 7.40$ (dd, $J=4.0,8.4 \mathrm{~Hz}, 1 \mathrm{H}), 1.34$ (s, 9H); ${ }^{13} \mathrm{C}$ NMR (100 MHz, $\left.\mathrm{CDCl}_{3}\right) \delta 166.3,150.3,146.6,144.7,136.1$, $135.9,135.2$, 132.8, 131.1, 129.0, 128.6, 128.5, 127.1, 125.9, 125.8, 121.3, 53.9, 30.6

(E)-3-(tert-butylimino)-6-methyl-2-(quinolin-8-yl)isoindolin-1one (3s)

Yellow solid; (73.8 mg, 86\% ); ${ }^{1} \mathrm{H}$ NMR (400 MHz, $\left.\mathrm{CDCl}_{3}\right) \delta$ 8.85 (dd, $J=1.6,4.0 \mathrm{~Hz}, 1 \mathrm{H}$ ), 8.20 (d, $J=8.4 \mathrm{~Hz}, 1 \mathrm{H}$ ), 8.00 (d, $J$ $=8.0 \mathrm{~Hz}, 1 \mathrm{H}), 7.88(\mathrm{~d}, J=6.8 \mathrm{~Hz}, 2 \mathrm{H}), 7.72(\mathrm{dd}, J=1.6,7.2 \mathrm{~Hz}$, $1 \mathrm{H}), 7.65(\mathrm{t}, J=7.2 \mathrm{~Hz}, 1 \mathrm{H}), 7.54$ (d, $J=7.6 \mathrm{~Hz}, 1 \mathrm{H}), 7.38$ (dd, $J$ $=4.0,8.0 \mathrm{~Hz}, 1 \mathrm{H}), 2.55(\mathrm{~s}, 3 \mathrm{H}), 1.36(\mathrm{~s}, 9 \mathrm{H}) ;{ }^{13} \mathrm{C}$ NMR $(100$ $\left.\mathrm{MHz}, \mathrm{CDCl}_{3}\right) \delta 167.7,150.2,147.9,145.0,141.8,136.0,134.5$, 133.2, 133.0, 131.2, 129.0, 128.3, 127.2, 125.9, 124.3, 121.2, 53.6, 30.5, 21.6

(E)-3-(tert-butylimino)-2-(quinolin-8-yl)-2,3-dihydro-1Hbenzo[f] isoindol-1-one (3t)

Yellow solid; (58.8 mg, 62\% ); ${ }^{1} \mathrm{H}$ NMR (400 MHz, $\left.\mathrm{CDCl}_{3}\right) \delta$ 8.86 (dd, $J=1.6,4.0 \mathrm{~Hz}, 1 \mathrm{H}), 8.57$ (d, $J=4.4 \mathrm{~Hz}, 2 \mathrm{H}), 8.19$ (dd, $J=2.0,8.4 \mathrm{~Hz}, 1 \mathrm{H}), 8.07-8.11$ (m, 2H), 7.89 (dd, $J=1.2,8.4 \mathrm{~Hz}$, $1 \mathrm{H}), 7.80$ (dd, $J=1.2,7.2 \mathrm{~Hz}, 1 \mathrm{H}), 7.66-7.70$ (m, 3H), 7.38 (dd, $J=4.0,8.0 \mathrm{~Hz}, 1 \mathrm{H}), 1.46$ (s, 9H); ${ }^{13} \mathrm{C} \mathrm{NMR}\left(100 \mathrm{MHz}, \mathrm{CDCl}_{3}\right) \delta$ 167.4, 150.2, 147.4, 144.8, 136.1, 135.1, 133.8, 133.3, 131.1, $130.8,129.9,129.5,129.1,128.5,128.4,128.4,128.3,126.0$, 125.9, 124.3, 121.3, 53.6, 30.4.

\section{(E)-4-(tert-butylimino)-5-(quinolin-7-yl)-4,5-dihydro-6H-thieno} [2,3-c]pyrrol-6-one (3u)

Yellow liquid; (25.2 mg, 30\% ); ${ }^{1} \mathrm{H}$ NMR (400 MHz, $\left.\mathrm{CDCl}_{3}\right) \delta$ 8.89 (dd, $J=1.6,4.0 \mathrm{~Hz}, 1 \mathrm{H}), 8.21$ (dd, $J=1.2,8.0 \mathrm{~Hz}, 1 \mathrm{H}$ ), 7.90 (dd, $J=1.2,6.8 \mathrm{~Hz}, 1 \mathrm{H}$ ), 7.76 (d, $J=4.8 \mathrm{~Hz}, 1 \mathrm{H}$ ), 7.72 (dd, $J=1.2,9.2 \mathrm{~Hz}, 1 \mathrm{H}), 7.66(\mathrm{t}, J=7.6 \mathrm{~Hz}, 1 \mathrm{H}), 7.53(\mathrm{~d}, J=5.2 \mathrm{~Hz}$, $1 \mathrm{H}), 7.42$ (dd, $J=4.0,8.4 \mathrm{~Hz}, 1 \mathrm{H}), 1.35$ (s, 9H); ${ }^{13} \mathrm{C}$ NMR $(100$ $\left.\mathrm{MHz}, \mathrm{CDCl}_{3}\right) \delta 162.6,150.3,145.9,145.1,141.5,139.8,136.1$, 134.6, 132.7, 131.4, 129.1, 128.4, 125.9, 125.2, 121.3, 54.2, 30.5.

\section{Acknowledgments}

Financial support from the National Science Foundation of China (NSFC) (grant number 21462021) and Scientific Research Fund of Jiangxi Provincial Education Department (grant number GJJ14238) are gratefully acknowledged.

\section{References and notes}

1. For reviews, see: (a) Dömling, A.; Ugi, I. Angew. Chem. Int. Ed. 2000, 39, 3168-3210. (b) Zhu, J. P.; Eur. J. Org. Chem. 2003, 1133-1144. (c) Nair, V.; Rajesh, C.; Vinod, A. U.; Bindu, S; Sreekanth, A. R.; Mathen, J. S.; Balagopal, L. Acc. Chem. Res. 2003, 36, 899-907. (d) Dömling, A. Chem. Rev. 2006, 106, 17-89. (e) El Kaim, L.; Grimaud, L. Tetrahedron 2009, 65, 2153-2171. (f) Sadjadi, S.; Heravi, M. M. Tetrahedron 2011, 67, 2707-2752. (g) van Berkel, S. S.; Bogels, B. G. M.; Wijdeven, M. A.; Westermann, B.; Rutjes, F. P. J. T. Eur. J. Org. Chem. 2012, 3543-3559.
2. (a) Gulevich, A. V.; Zhdanko, A. G.; Orru, R. V. A.; Nenajdenko, V. G. Chem. Rev. 2010, 110, 5235-5331. (b) Fayol, A.; Zhu, J.; Angew. Chem., Int. Ed. 2002, 41, 3633-3635. (c) Bonne, D.; Dekhane, M.; Zhu, J. Angew. Chem., Int. Ed. 2007, 46, 2485-2488. (e) Yue, T.; Wang, M.; Wang, D.; Masson, G.; Zhu, J. Angew. Chem., Int. Ed. 2009, 48, 6717-6721. (f) Buyck, T.; Wang, Q.; Zhu, J. Angew. Chem., Int. Ed. 2013, 52, 12714-12718.

3. Recent reviews, see: (a) Zhang, B.; Studer, A. Chem. Soc. Rev. 2015, 44, 3505-3521. (b) Lygin, A. V. Meijere, A. D. Angew. Chem. Int. Ed. 2010, 49, 9094-9124. (c) Sharma, U. K.; Sharma, N.; Vachhani, D. D.; Van der Eycken, E. V. Chem. Soc. Rev. 2015, 44, 1836-1860.

4. Recent reviews for the isocyanide insertion: (a) Qiu,G.; Ding, Q.; Wu, J. Chem. Soc. Rev. 2013, 42, 5257-5269. (b) Vlaar, T.; Maes, B. U. W.; Ruijter, E.; Orru. R. V. A. Angew. Chem. Int. Ed. 2013, 52, 7084-7097. (c) Lang, S.; Chem. Soc. Rev. 2013, 42, 4867-4880. (d)Lauzon, J. M. P.; Schafer, L. L. Dalton Trans. 2012, 41, 1153911550.

5. (a) Saluste, C.; Whitby, R.; Furber, M. Angew. Chem., Int. Ed. 2000, 39, 4156-4158. (c) Baelen, G.; Kuijer, S.; Rýček, L.; Sergeyev, S.; Janssen, E.; de Kanter, F.; Maes, B.; Ruijter, E.; Orru, R. Chem.-Eur. J. 2011, 17, 15039-15044. (d) Jiang, H.; Liu, B.; Li, Y.; Wang, A.; Huang, H. Org. Lett. 2011, 13, 10281031.

6. (a) Wang, Y.; Wang, H.; Peng, J.; Zhu, Q. Org. Lett. 2011, 13, 4604-4607. (b) Vlaar,T.; Cioc, R. C.; Mampuys, P.; Maes, B. U. W.; Orru, R. V. A.; Ruijter, E. Angew. Chem. Int. Ed., 2012, 51, 13058-13061. (c) Xu, S.; Huang, X.; Hong, X., Xu, B. Org. Lett., 2012, 14, 4614-4617. (d) Jones, W.; Kosar, W. J. Am. Chem. Soc. 1986, 108, 5640-5641. (e) Fukumoto, Y.; Hagihara, M.; Kinashi, F.; Chatani. N. J. Am. Chem. Soc. 2011, 133, 10014-10017.

7. (a) Takamatsu, K.; Hirano, K.; Miura, M. Org. Lett. 2015, 17, 4066-4069. (b) Zhu, T.; Wang, S.; Tao, Y.; Wei, T.; Ji, S. Org. Lett. 2014, 16, 1260-1263.

8. (a) Cai, X.; Xie, B. Curr. Org. Chem. 2015, 19, 121-150. (b) Hyster, T. K. Catal. Lett. 2015, 145, 458-467. (c) Tilly, D.; Dayaker, G.; Bachu, P. Catal. Sci. Technol. 2014, 4, 2756-2777. (d) Yoshikai, N. Bull. Chem. Soc. Jpn. 2014, 87, 843-857. (f) Gao, K.; Yoshikai, N. Acc. Chem. Res. 2014, 47, 1208-1219. (g) Ackermann, L. J. Org. Chem. 2014, 79, 8948-8954. (h) Yoshikai, N. Synlett 2011, 2011, 1047-1051. (i) Kulkarni, A. A.; Daugulis, O. Synthesis 2009, 4087-4109.

9. For selected recent examples, see: (a) Zhao, D.; Kim, J. H.; Stegemann, L.; Strassert, C. A.; Glorius, F. Angew. Chem., Int. Ed. 2015, 54, 4508-4511. (b) Hummel, J. R.; Ellman, J. A. J. Am. Chem. Soc. 2015, 137, 490-498. (c) Fallon, B. J.; Derat, E.; Amatore, M.; Aubert, C.; Chemla, F.; Ferreira, F.; PerezLuna, A.; Petit, M. J. Am. Chem. Soc. 2015, 137, 2448-2451. (d) Li, J.; Ackermann, L. Angew. Chem., Int. Ed. 2015, 54, 3635-3638. (e) Grigorjeva, L.; Daugulis, O. Angew. Chem., Int. Ed. 2014, 53, 10209-10212. (f) Yu, D.; Gensch, T.; de Azambuja, F.; VasquezCespedes, S.; Glorius, F. J. Am. Chem. Soc. 2014, 136, 17722-17725. (g) Ikemoto, H.; Yoshino, T.; Sakata, K.; Matsunaga, S.; Kanai, M. J. Am. Chem. Soc. 2014, 136, 5424-5431. (h) Gao, K.; Yoshikai, N. J. Am. Chem. Soc. 2013, 135, 9279-9282. (i) Yoshino, T.; Ikemoto, H.; Matsunaga, S.; Kanai, M. A. Angew. Chem., Int. Ed. 2013, 52, 2207-2211. (j) Yao, T.; Hirano, K.; Satoh, T.; Miura, M. Angew. Chem., Int. Ed. 2012, 51, 775-779. (k) Song, W.; Ackermann, L. Angew. Chem., Int. Ed. 2012, 51, 8251-8254. (l) Chen, Q.; Ilies, L.; Nakamura, E.-I. J. Am. Chem. Soc. 2011, 133, 428-429. (m) Li, B.; Wu, Z.; Gu, Y.; Sun, C.; Wang, B.; Shi, Z. Angew. Chem., Int. Ed. 2011, 50, 1109-1113. (n) Gao, K.; Lee, P. S.; Fujita, T.; Yoshikai, N. J. Am. Chem. Soc. 2010, 132, 12249-12251. For the first example of cobalt-catalyzed $\mathrm{C}-\mathrm{H}$ functionalization, see: (o) Murahashi, S. J. Am. Chem. Soc. 1955, 77, 6403-6404.

10. (a) Zhang, L.; Hao, X.; Zhang, S.; Liu, Z.; Zheng, X.; Gong, J.; Niu, J.; Song, M. Angew. Chem., Int. Ed. 2015, 54, 272-275. (b) Wu, X.; Yang, K.; Zhao, Y.; Sun, H.; Li, G.; Ge, H. Nat. Commun. 2015, 6, 6462. (c) Sun, B.; Yoshino, T.; Matsunaga, S.; Kanai, M. Chem. Commun. 2015, 51, 4659-4661. (d) Patel, P.; Chang, S. ACS Catal. 2015, 5, 853-858. (e) Lu, H.; Li, C.; Jiang, H.; Lizardi, C.; Zhang, X. P. Angew. Chem., Int. Ed. 2014, 53, 7028-7032. (f) Jin, L.; Lu, H.; Cui, Y.; Lizardi, C. L.; Arzua, T. N.; Wojtas, L.; Cui, X.; Zhang, X. P. Chem. Sci. 2014, 5, 2422-2427. (g) Zardi, P.; Intrieri, D.; Caselli, A.; Gallo, E. J. Organomet. Chem. 2012, 716, 269-274. (h) Kim, J.; Cho, S.; Joseph, J.; Chang, S. Angew. Chem., Int. Ed. 2010, 
49, 9899-9903. (i) Lu, H.; Jiang, H.; Wojtas, L.; Zhang, X. P. Angew. Chem., Int. Ed. 2010, 49, 10192-10196. (j) Cenini, S.; Gallo, E.; Penoni, A.; Ragaini, F.; Tollari, S. Chem. Commun. 2000, 2265-2267.

11. Grigorjeva, L.; Daugulis, O. Angew. Chem., Int. Ed. 2014, 53, 10209-10212.

12. W.Y. Hao, J. Tian, W. Li, R. Y. Shi, Z. L. Huang, A.W. Lei, Chem.Asian J. 2016, 11,1664-1667.

13. (a) T.-H. Zhu, S.-Y. Wang, G.-N. Wang, Chem.-Eur. J. 2013, 19, 5850-5853.(b) T.-H. Zhu, S.-Y. Wang, T.-Q. Wei, S.-J. Ji, Adv. Synth. Catal. 2015, 357, 823-828; (c) T.-H. Zhu, X.-P. Xu, J. -J. Cao, T.-Q. Wei, S.-Y. Wang, S.-J. Ji, Adv. Synth. Catal. 2014, 356, 509-518.
14. (a) Hao, W. Y.; Jiang, Y. Y.; Cai, M. Z. J. Org. Chem., 2014, 79, 3634-3640. (b) Hao, W. Y.; Zeng, J. B.; Cai, M. Z. Chem. Commun., 2014, 50, 11686-11689. (c) Hao, W. Y.; Huang, J.; Jie, S.; Cai, M. Z. Eur. J. Org. Chem. 2015, 6655.

15. (a) Zhang, J.; Chen, H.; Lin, C.; Liu, Z.; Wang, C.; Zhang, Y. J. Am. Chem. Soc. 2015, 137, 12990-12996. (b) Ma, W.; Ackermann, L. ACS Catal. 2015, 5, 2822-2825. (c) Zhang, L.; Hao, X.; Liu, Z.; Zheng, X.; Zhang, S.; Niu, J.; Song, M.; Angew.Chem. Int. Ed. 2015, 54, 10012-10015.

Click here to remove instruction text... 


\section{Graphical Abstract}

To create your abstract, type over the instructions in the template box below.

Fonts or abstract dimensions should not be changed or altered.

\section{An efficient synthesis of iminoisoindolione} derivatives by cobalt-catalyzed oxidative

Leave this area blank for abstract info. isocyanide insertion

Feihua Zou, Xuwen Chen, Wenyan Hao*

College of Chemistry \&Chemical Engineering, Jiangxi Normal University, Nanchang, 330022, People's Repulic of China

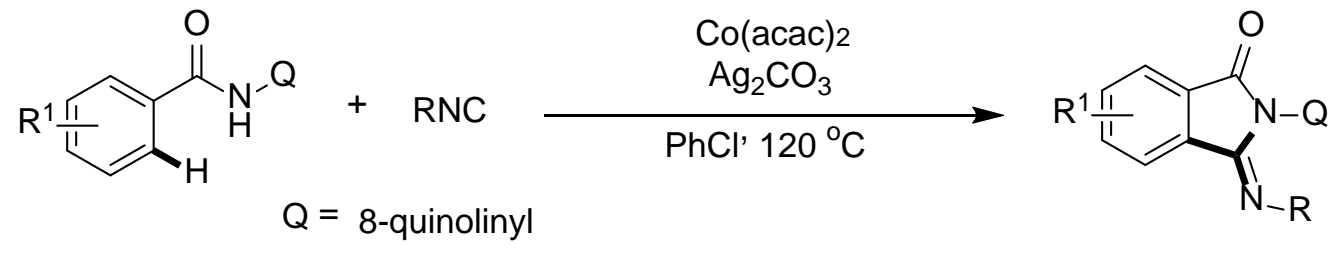

